\title{
Anti-CD45 Monoclonal Antibody BC8
}

National Cancer Institute

\section{Source}

National Cancer Institute. Anti-CD45 Monoclonal Antibody BC8. NCI Thesaurus. Code C1961.

A murine IgG1 anti-CD45 monoclonal antibody (MoAb) with immunotherapeutic activity. CD45 antigen, a receptor protein-tyrosine phosphatase essential for T cell development and lymphocyte activation, is expressed on virtually all leukocytes. MoAb BC8 has specificity for hematopoietic tissues and may be used in targeted immunotherapy. 